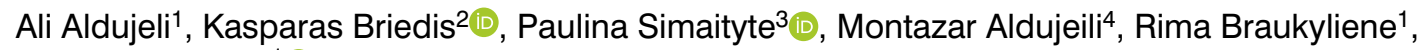
Ramunas Unikas ${ }^{1}$ (1)

${ }^{1}$ Department of Cardiology, Medical Academy, Lithuanian University of Health Sciences, Kaunas, Lithuania

${ }^{2}$ Liverpool Heart and Chest Hospital, NHS foundation trust, Liverpool, United Kingdom

${ }^{3}$ Faculty of Medicine, Lithuanian University of Health Sciences, Kaunas, Lithuania

${ }^{4}$ Medical Academy, University of Brescia, Brescia, Italy

\title{
The influence of obesity on in-hospital clinical outcomes after recanalisation of chronic total occlusions
}

\section{Corresponding author:}

Ali Aldujeli, Department of Cardiology,

Medical Academy, Lithuanian

University of Health Sciences,

Kaunas, Lithuania,

e-mail: Ali.Aldujeli@kaunoklinikos.It

Medical Research Journal 2020;

Volume 5, Number 1, 19-22

10.5603/MRJ.a2020.0003

Copyright $\odot 2020$ Via Medica

ISSN 2451-2591

\begin{abstract}
Objectives: Recanalisation of chronic total occlusions (CTOs) in interventional cardiology is one of the most challenging and complex procedures. Currently, no data are available about the impact of BMI on success rates among CTO patients undergoing percutaneous coronary intervention. The aim of this study was to investigate the impact that BMI has on success rates, complications, and procedure characteristics among a large group of СТO patients who underwent percutaneous coronary intervention.

Methods: The present study retrospectively included 420 patients who underwent percutaneous coronary intervention for at least to one chronic total occlusion. Patients were subdivided into groups according to their BMI. BMI groups were based on the World Health Organization's definitions: From 18.5 to $24.9 \mathrm{~kg} / \mathrm{m}^{2}=$ Normal weight; From 25 to $29.9 \mathrm{~kg} / \mathrm{m}^{2}$ = Overweight; From 30 to $34.9 \mathrm{~kg} / \mathrm{m}^{2}$ = Obesity; Greater than or equal to $35 \mathrm{~kg} / \mathrm{m}^{2}=$ Very obese. In order to subdivide the patients according to the complexity of the CTO-PCI, the Japanese-CTO (J-CTO) score was used prior to the interventions. Statistical analyses were performed using the SPSS 20.0 software. The value of $p<0.05$ was considered as statistically significant.

Results: There was a significant difference in procedure duration, volume of used contrast medium and other procedural characteristics in higher BMI categories. There was no statistically significant difference across all body mass index categories in terms of procedure success, complication rates, and outcomes $(p>0.05)$. Conclusion: This retrospective study indicates that BMI has no impact on in-hospital outcomes in patients with chronic total occlusion after percutaneous coronary intervention.
\end{abstract}

Key words: obesity, chronic total occlusion, percutaneous coronary intervention, coronary artery disease Med Res J 2020; 5 (1): 19-22

\section{Introduction}

Recanalisation of chronic total occlusions (CTOs) in interventional cardiology is one of the most challenging and complex procedures [1]. Coronary artery CTOs can be identified in around one in every five patients who show clinical indications for coronary angiography [2]. Recanalisation of CTOs is indicated in cases of progressing angina symptoms, with evidence of significant myocardial ischaemia [3]. After recanalisation in these patients, an increase in left ventricular function can be expected [4]. Due to the emerging methods in interventional cardiology and the use of new СТО equipment, СТО recanalisation success rates have increased steadily in recent years
[5]. Moreover, coronary artery bypass grafts (CABG) can be avoided, which can result in lower complication rates [6].

Worldwide obesity levels have increased in recent decades [7]. Obesity and, in particular, severe obesity are linked to a heightened risk of cardiovascular disease (CVD) [8] because BMI is a strong predictor of cardiovascular mortality [9].

Despite the heightened risks, the in-hospital outcomes after СTO percutaneous coronary interventions $(\mathrm{PCl})$ remain unstudied in this population. Accordingly, this study retrospectively evaluates the impact of $\mathrm{BMI}$ on success and complication rates, as well as procedure characteristics among a large group of СTO patients undergoing $\mathrm{PCl}$. 


\section{Method and materials}

\section{Study population}

A total of 420 patients who underwent CTO-PCl at the Lithuanian University of Health Sciences of Kaunas were included in this study. Criteria for inclusion were a positive test for angina pectoris and/or positive result for functional ischaemia from a stress echocardiography or magnetic resonance imaging test. Patients were subdivided into groups according to their BMI. BMI groups were based on the World Health Organisation's definitions [10]:

- From 18.5 to $24.9 \mathrm{~kg} / \mathrm{m} 2$ = Normal weight

- From 25 to $29.9 \mathrm{~kg} / \mathrm{m} 2$ = Overweight

- From 30 to $34.9 \mathrm{~kg} / \mathrm{m} 2$ = Obesity

- Greater than or equal to $35 \mathrm{~kg} / \mathrm{m} 2=$ Very obese CTO-PCl procedure

Either radial or femoral access was chosen according to operator choice. The diameter of guiding catheters was either 6 or 7F. Heparin was administered at the start of interventions, guided by a clotting time of greater than 300 seconds, in order to prevent thromboembolic complications. All PCls were performed via anterograde approach. In most cases, dual injections were made to define the length of the lesion and for the conformation that the $\mathrm{PCl}$ guide wire is in the true lumen. In case of femoral access, the puncture site was sealed using manual compression or various vascular closure devices.

Coronary wires ranged from tapered polymer softtip guide wires at the start to super-stiff guide wires at the end. In almost all cases, drug-eluting stents were implanted in the occluded segments. Stent apposition and expansion was optimised by post-dilatation.

In order to subdivide the patients according to the complexity of the CTO-PCI, the Japanese-CTO (J-CTO) score was used prior to the interventions [11]. This combined the following parameters:

- Lesion classification degree.

- Bending in excess of 45 degrees in the CTO segment.

- Blunt proximal cap.

- Occluded segment length (> $20 \mathrm{~mm}$ ).

- Previously failed recanalisation.

The definition of success in the procedure was a restoration of TIMI grade 3 flow and recanalisation of CTO. Each patient undergoing the procedure had a composite safety endpoint evaluation. This summarised severe complications including stroke, cardiac tamponade, vascular complications, or in-hospital death.

\section{Statistical analysis}

Continuous variables are shown as median, mean \pm standard deviation, and minimum-maximum.
Category variables are presented as percentages and frequencies. Normal distribution was tested for using the Shapiro-Wilk test. Mann-Whitney $U$ test or the Kruskal-Wallis test were used as appropriate for continuous variables and the Fisher exact test for categorical variables.

This study was given approval by the Research Ethics Committee of the Government of Kaunas (Lithuania). The study complies with the principles laid down in the Declaration of Helsinki, adopted by the $18^{\text {th }}$ World Medical Assembly, Helsinki, Finland in June 1964 and recently amended at the $59^{\text {th }}$ World Medical Assembly, Seoul, Korea in October 2008.

\section{Results}

\section{Demographics}

Males (62\%) made up the majority of patients, with a mean age of 68 years ( \pm 5.4 years). Male patients were younger than female patients $(67 \pm 7.1$ years vs. $72.2 \pm 4.4$ years; $p<0.001$ ). Mean BMI was $26.8 \mathrm{~kg} / \mathrm{m}^{2}\left( \pm 6.5 \mathrm{~kg} / \mathrm{m}^{2}\right)$. From the 420 patients the following BMls were recorded:

- Normal weight - $42(10 \%)$

- Overweight - $252(60 \%)$

- Obese - 100 (23.8\%)

- Very obese - 26 (6.2\%)

\section{Risk factors}

There was a positive correlation between rising BMI categories and cardiovascular risk elements such as arterial hypertension, diabetes mellitus, and family history of coronary artery disease. Compared to patients with a BMI greater than $25 \mathrm{~kg} / \mathrm{m}^{2}$, patients with normal weight showed higher HDL cholesterol $(p<0.05)$ and lower triglycerides $(p<0.05)$. Very obese patients had a higher incidence of diabetes mellitus compared to other subgroups ( $p=0.015)$. Overweight patients had a higher rate of chronic obstructive pulmonary disease and lower peripheral arterial disease prevalence.

\section{Procedural characteristics}

Procedural and CTO characteristics are displayed in Table 1. All BMI categories had a similar J-CTO score $(p>0.05)$. The amount of contrast medium used increased as the BMI increased $(p=0.012)$. The fluoroscopy time tended to increase proportionally with rising BMI $(p=0.03)$. Procedure duration of normal patients was lower as compared to patients with non-normal BMls $(p=0.024)$. Success rates were similar across all BMl categories ( $p=0.622$; Fig. 1$)$. 
Table 1. Procedural and angiographic characteristics

\begin{tabular}{lccccc}
\hline & Normal & Overweight & Obese & Very obese & P \\
\hline CTO in LAD & $28.6 \%(12)$ & $28.6 \%(72)$ & $32 \%(32)$ & $34 \%(9)$ & 0.65 \\
CTO in LCX & $23.8 \%(10)$ & $31.7 \%(80)$ & $18 \%(18)$ & $27 \%(7)$ & \\
CTO in RCA & $47.6 \%(20)$ & $39.7 \%(100)$ & $50 \%(50)$ & $38.5 \%(10)$ & \\
1-vessel disease & $26.2 \%(11)$ & $20.7 \%(52)$ & $35 \%(35)$ & $26.9 \%(7)$ & 0.84 \\
2-vessel disease & $28.6 \%(12)$ & $31.7 \%(80)$ & $29 \%(29)$ & $26.9 \%(7)$ & \\
3-vessel disease & $45.2 \%(19)$ & $47.6 \%(120)$ & $36 \%(36)$ & $46.2 \%(12)$ & \\
J-CTO score $\geq 3$ & $59.5 \%(25)$ & $65 \%(164)$ & $63 \%(63)$ & $57.7 \%(15)$ & 0.58 \\
Amount of contrast $(\mathrm{ml})$ & $220(80-320)$ & $260(100-500)$ & $290(120-650)$ & $350(200-800)$ & 0.012 \\
Procedure time $(\mathrm{min})$ & $60.5(30-109)$ & $126.1(60-190)$ & $160.7(60-230)$ & $190.2(90-260)$ & 0.024 \\
Fluoroscopy time $(\mathrm{min})$ & $25.6(6-40)$ & $30.1(12-60)$ & $35.4(15-94)$ & $40.2(20-95)$ & 0.03 \\
\hline
\end{tabular}

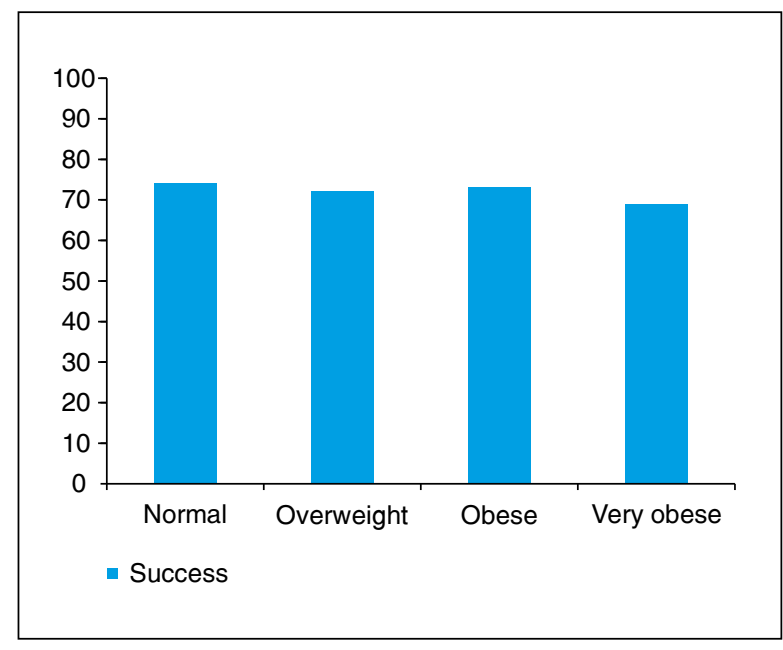

Figure 1. Percentage of success depending on BMI

\section{Complication rates}

There was no statistically significant difference in procedural complications, which rarely occurred $(p=0.12)$. There were no acute skin lesions witnessed as radiation doses increased with higher BMl categories $(p<0.001)$. The complications that did occur were mostly vascular. In three cases cardiac tamponade occurred. Similarly, three patients were diagnosed with acute stroke after CTO-PCI. No in-hospital deaths were recorded (Fig. 2).

\section{Discussion}

The study highlights some important aspects. Firstly, successful procedures and complications in the hospital proved to be independent of BMl categories. This finding seems quite remarkable, given that we are already aware of overweight and obese patients from Western countries having a higher likelihood of getting

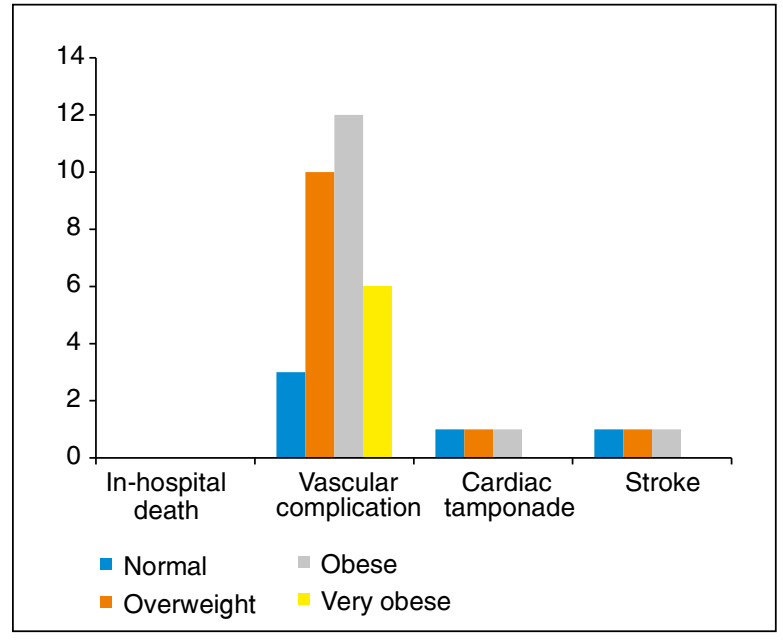

Figure 2. In-hospital outcomes

a wound infection following CABG [12]. This suggests that CTO-PCls might be a safer alternative to CABG in very obese patients.

Secondly, patients in higher BMI categories had an increase in prevalence of cardiovascular risk factors, such as arterial hypertension, diabetes mellitus, dyslipidaemia, triglyceride, and HDL-cholesterol levels. These co-morbidities are related to the metabolic syndrome and are in line with current literature on the subject [13].

Thirdly, there was a significant difference in the procedure duration, volume of used contrast medium, and other procedural characteristics in higher BMI categories. Obese patients had higher doses of radiation $(p<0.001)$ and longer procedure times $(p=0.026)$. Dermal ulcerations could be a serious consequence of such procedures, although there are no supporting data from our study due to the short follow-up period. Nevertheless, it has been proven by Lia et al. that dermal ulcerations can occur during PCls, especially in CTOs because there is an increase of these lesions with higher 
radiation doses [14]. Thus, given a longer follow-up period, higher radiation-associated complication rates could be expected in obese patients.

There are divergent opinions on the relationship between BMI and outcomes in CAD patients. The 'obesity paradox' is that despite obesity being a strong CVD development risk factor, mortality rates for obese and very obese patients are low. One possible reason for this paradox is that obese patients may be observed more and therefore receive faster medical intervention. Also, obese patients tend to be younger when the acute cardiovascular event occurs. This could imply that there is benefit regarding age [15].

A Korean study published in 2012 revealed that the relation between $\mathrm{BMI}$ and mortality was $U$-shaped, with the nadir among overweight or obese patients, and underweight and normal-weight patients having the highest risk [16]. Their definition of obese, however, was a BMI greater than $25 \mathrm{~kg} / \mathrm{m}^{2}$, not $30 \mathrm{~kg} / \mathrm{m}^{2}$, as in our study. Also, half of the patients in their study had first generation drug-eluting stents (DES) implanted. In the long term, overweight patients, particularly men, had a reduced mortality rate after CTO-PCI, according to a study conducted in the USA in 2010 [17]. The patients in their study, however, were older than those in our study.

Contrasting trials, in line with our own, have failed to indicate a relationship between $\mathrm{BMI}$ and $\mathrm{PCl}$ survival. For instance, data presented from over 5000 patients led Diletti et al. to conclude that, following coronary artery interventions, BMI had no impact on the long-term clinical outcomes [18].

Another method of treatment considered as a substitute for CTO patients could be CABG. Some data indicate that mortality was not increased by obesity in the short-term perspective; however, this is associated with an increase in late mortality rates. [19].

We can demonstrate that there is no dependence between in-hospital clinical events and BMI. This is in contrast to previous data that suggested that very obese and lean patients are at greater risk following $\mathrm{PCI}$ [20].

\section{Conclusion}

Our ex post facto study indicates that obesity has no impact on in-hospital outcomes in CTO-PCl patients.

\section{Conflict of interest statement: None}

\section{References}

1. Konstantinidis N, Pighi M, Dogu Kilic I, et al. New Advances in Chronic Total Occlusions. Interv Cardiol. 2014; 9(3): 208-212, doi 10.15420/icr.2014.9.3.208, indexed in Pubmed: 29588804.
2. Fefer P, Knudtson ML, Cheema AN, et al. Current perspectives on coronary chronic total occlusions: the Canadian Multicenter Chronic Total Occlusions Registry. J Am Coll Cardiol. 2012; 59(11): 991-997, doi: 10.1016/i.jacc.2011.12.007, indexed in Pubmed: 22402070.

3. Rawlins J, Wilkinson J, Curzen N. Evidence for Benefit of Percutaneous Coronary Intervention for Chronically Occluded Coronary Arteries (CTO) - Clinical and Health Economic Outcomes. Interv Cardiol. 2014; 9(3): 190-194, doi: 10.15420/icr.2014.9.3.190, indexed in Pubmed: 29588801.

4. Chimura M, Yamada S, Yasaka Y, et al. Improvement of left ventricular function assessment by global longitudinal strain after successful percutaneous coronary intervention for chronic total occlusion. PLoS One. 2019; 14(6): e0217092, doi: 10.1371/journal.pone.0217092, indexed in Pubmed: 31188846.

5. Gülker JE, Bansemir L, Klues HG, et al. Chronic total coronary occlusion recanalization: Current techniques and new devices. J Saudi Heart Assoc. 2017; 29(2): 110-115, doi: 10.1016/j.jsha.2016.08.003, indexed in Pubmed: 28373785.

6. Shah A. Chronic Total Occlusion Coronary Intervention: In Search of a Definitive Benefit. Methodist Debakey Cardiovasc J. 2018; 14(1): 50-59, doi: 10.14797/mdcj-14-1-50, indexed in Pubmed: 29623172.

7. Agha M, Agha R. The rising prevalence of obesity: part A: impact on public health. Int J Surg Oncol (N Y). 2017; 2(7): e17, doi: 10.1097/IJ9.0000000000000017, indexed in Pubmed: 29177227.

8. Akil L, Ahmad HA. Relationships between obesity and cardiovascular diseases in four southern states and Colorado. J Health Care Poor Underserved. 2011; 22(4 Suppl): 61-72, doi: 10.1353/hpu.2011.0166, indexed in Pubmed: 22102306.

9. Chen Yu, Copeland WK, Vedanthan R, et al. Association between body mass index and cardiovascular disease mortality in east Asians and south Asians: pooled analysis of prospective data from the Asia Cohort Consortium. BMJ. 2013; 347: f5446, doi: 10.1136/bmj.f5446, indexed in Pubmed: 24473060

10. Bucholz EM, Rathore SS, Reid KJ, et al. Body mass index and mortality in acute myocardial infarction patients. Am J Med. 2012; 125(8): 796-803, doi: 10.1016/j.amjmed.2012.01.018, indexed in Pubmed: 22483510.

11. Christopoulos G, Wyman RM, Alaswad K, et al. Clinical Utility of the Japan-Chronic Total Occlusion Score in Coronary Chronic Total Occlusion Interventions: Results from a Multicenter Registry. Circ Cardiovasc Interv. 2015; 8(7): e002171, doi: 10.1161/CIRCINTERVENTIONS.114.002171, indexed in Pubmed: 26162857.

12. Kotnis-Gąska A, Mazur P, Olechowska-Jarząb A, et al. Sternal wound infections following cardiac surgery and their management: a single-centre study from the years 2016-2017. Kardiochir Torakochirurgia Pol. 2018; 15(2): 79-85, doi: 10.5114/kitp.2018.76472, indexed in Pubmed: 30069187.

13. Kadakia MB, Fox CS, Scirica BM, et al. Central obesity and cardiovascular outcomes in patients with acute coronary syndrome: observations from the MERLIN-TIMI 36 trial. Heart. 2011; 97(21): 1782-1787, doi: 10.1136/heartjnl-2011-300231, indexed in Pubmed: 21865203.

14. Lai CC, Wei KC, Chen WY, et al. Risk Factors For Radiation-Induced Skin Ulceration in Percutaneous Coronary Interventions of Chronic Total Occluded Lesions: A 2-Year Observational Study. Sci Rep. 2017; 7(1): 8408, doi: 10.1038/s41598-017-08945-4, indexed in Pubmed: 28814768.

15. Patel N, Elsaid O, Shenoy A, et al. Obesity paradox in patients undergoing coronary intervention: A review. World J Cardiol. 2017; 9(9): 731-736, doi: 10.4330/wjc.v9.i9.731, indexed in Pubmed: 29081905.

16. Won KB, Yoon HJ, Lee SG, et al. Comparison of long-term mortality according to obesity in patients with successful percutaneous chronic total occlusion interventions using drug-eluting stents. Catheter Cardiovasc Interv. 2018; 91(4): 710-716, doi: 10.1002/ccd.27110, indexed in Pubmed: 28976619.

17. Stähli BE, Gebhard C, Gick M, et al. Impact of body mass index on long-term mortality in women and men undergoing percutaneous coronary intervention for chronic total occlusion. Int J Cardiol. 2016; 224: 305-309, doi: 10.1016/i.ijcard.2016.09.057, indexed in Pubmed: 27665402.

18. Diletti R, Garcia-Garcia HM, Bourantas C, et al. Impact of body mass index on long-term clinical outcomes after second-generation drug eluting stent implantation: Insights from the international global RESOLUTE program. Catheter Cardiovasc Interv. 2015; 85(6): 952-958, doi: 10.1002/ccd.25828, indexed in Pubmed: 25689692.

19. Hällberg V, Kataja M, Lahtela J, et al. W-CABG Study Group. Obesity paradox disappears in coronary artery bypass graft patients during 20year follow-up. Eur Heart J Acute Cardiovasc Care. 2017; 6(8): 771-777, doi: 10.1177/2048872616633844, indexed in Pubmed: 26912911.

20. Benderly M, Boyko V, Goldbourt U. Relation of body mass index to mortality among men with coronary heart disease. Am J Cardiol. 2010; 106(3): 297-304, doi: 10.1016/j.amjcard.2010.03.078, indexed in Pubmed: 20643236 